\title{
ASPECTOS ASSOCIADOS À PRODUÇÃO INTELECTUAL DOS PROGRAMAS DE PÓS-GRADUAÇÃO EM ADMINISTRAÇÃO PÚBLICA E DE EMPRESAS, CIÊNCIAS CONTÁBEIS E TURISMO
}

Gabriel Filipe Veloso da Costa, Universidade Federal de Viçosa, Campus Rio Paraíba.
Ney Paulo Moreira, Universidade Federal de Viçosa, $\frac{\underline{\text { Campus Rio Paraíba. }}}{\text { Campromoreira@ ufv.br }}$
Wagner Soares Bonfim Junior, Universidade Federal de Viçosa, Campus Rio Paraíba.

Wagner.fonfim@ufv.br

\section{RESUMO}

O objetivo do trabalho foi analisar a produção intelectual da pós-graduação brasileira da área de Administração Pública e de Empresas, Ciências Contábeis e Turismo, buscando identificar os fatores que influenciaram na produção científica desses programas no quadriênio 2013-2016. O estudo se baseia em pesquisa quantitativa, explicativa e ex post facto. Os dados da produção intelectual dos 182 programas de pós-graduação stricto sensu da área analisada foram coletados em planilhas disponibilizadas pela CAPES. Foi calculada a produção média dos programas e, posteriormente, empregou-se a análise de regressão múltipla para identificar os aspectos associados à produção intelectual. Os resultados apontaram existir grande heterogeneidade em relação à produção intelectual dos programas de pós-graduação analisados, principalmente ao considerar os níveis de publicação em periódicos do estrato A1. A pontuação média por docente permanente também revelou a expressiva disparidade existente no quantitativo de artigos publicados em periódicos. Analisando os aspectos relacionados à produção intelectual dos programas de pós-graduação, identificou-se que o tempo de funcionamento dos programas, a publicação de artigos em anais de eventos, bem como o percentual médio de discentes contemplados com bolsas de estudos apresentaram associação positiva com a produção média dos programas de pós-graduação. Por outro lado, o tamanho dos programas, representado pelo quantitativo de docentes e discentes, mostrou relação negativa com a produção média por docente. Observou-se ainda que os programas de pós-graduação vinculados às instituições privadas de ensino apresentaram maior produção intelectual, se comparados aos programas vinculados às instituições públicas. Conclui-se, portanto, que o estímulo à publicação de trabalhos em eventos acadêmicos, bem como o pagamento de bolsas aos discentes são ações que devem ser incentivadas, pois se mostraram capazes de contribuir com o desenvolvimento científico da área de Administração Pública e de Empresas, Ciências Contábeis e Turismo.

Palavras-chave: Avaliação, Produção científica, Qualis CAPES.

Data de recebimento: $24 / 10 / 2020$

Data do aceite de publicação: 15/04/2021

Data da publicação: 30/04/2021 


\title{
ASPECTOS ASSOCIADOS À PRODUÇÃO INTELECTUAL DOS \\ PROGRAMAS DE PÓS-GRADUAÇÃO EM ADMINISTRAÇÃO PÚBLICA E \\ DE EMPRESAS, CIÊNCIAS CONTÁBEIS E TURISMO
}

\section{ASPECTS ASSOCIATED WITH THE INTELLECTUAL PRODUCTION OF GRADUATE PROGRAMS IN PUBLIC AND BUSINESS ADMINISTRATION, ACCOUNTING AND TOURISM}

\author{
Gabriel Filipe Veloso da Costa, Universidade Federal de Viçosa, Campus Rio Paraíba. \\ Gabriel.filipe@ufv.br \\ Ney Paulo Moreira, Universidade Federal de Viçosa, Campus Rio Paraíba. \\ Ney.moreira@ufv.br \\ Wagner Soares Bonfim Junior, Universidade Federal de Viçosa, Campus Rio Paraíba. \\ Wagner.fonfim@ufv.br
}

\begin{abstract}
The purpose of this study was to analyze the intellectual production of Brazilian graduate courses in the area of Public and Business Administration, Accounting and Tourism, seeking to identify the factors that influenced the scientific production of these programs in the period from 2013 to 2016 . The study is based on quantitative, explanatory, and ex post facto research. The data on the intellectual production of 182 graduate programs in the analyzed area were collected in worksheets made available by CAPES. The average production of the programs was calculated and, later, multiple regression analysis was used to identify the aspects associated with intellectual production. The results showed that there is great heterogeneity in relation to the intellectual production of the analyzed graduate programs, especially when considering the levels of publication in journals of stratum A1. The average score per permanent professor also revealed the significant disparity in the number of papers published in journals. Analyzing the aspects related to the intellectual production of graduate programs, it was identified that the age of programs, the publication of papers in the annals of events and the average percentage of students contemplated with scholarships presented a positive association with the average production of graduate programs. However, the size of the programs, represented by the number of professors and students, showed a negative relationship with the average production per professor. It was also observed that graduate programs offered by private educational institutions showed greater intellectual production, when compared to programs offered by public institutions. The paper concludes that stimulating the publication of papers in academic events and the payment of scholarships to students are actions that should be encouraged, because they have been shown to contribute to the scientific development of the Public and Business Administration, Accounting and Tourism area.
\end{abstract}

Keywords: Evaluation, Scientific production, Qualis system of CAPES.

\section{INTRODUÇÃO}

A pós-graduação brasileira da área de Administração Pública e de Empresas, Ciências Contábeis e Turismo tem apresentado um expressivo crescimento no quantitativo de cursos sendo oferecidos. Segundo estatísticas da Coordenação de Aperfeiçoamento de Pessoal de Nível Superior [CAPES] (2019), desde o ano de 1998 o 


\section{ASPECTOS ASSOCIADOS À PRODUÇÃO INTELECTUAL DOS \\ PROGRAMAS DE PÓS-GRADUAÇÃO EM ADMINISTRAÇÃO PÚBLICA E \\ DE EMPRESAS, CIÊNCIAS CONTÁBEIS E TURISMO}

número de programas de pós-graduação stricto sensu (mestrado e doutorado) passou de 21 para 198 programas em 2018, representando um acréscimo de mais de 840\%.

Esse acréscimo no número de cursos de pós-graduação pode ser visto, a princípio, como uma melhoria no desenvolvimento científico da referida área. No entanto, pode-se questionar até que ponto esse crescimento não é apenas consequência da própria expansão do ensino superior do país, tendo em vista que a ampliação de vagas no ensino de graduação impulsiona também a demanda por profissionais qualificados com mestrado e doutorado para lecionarem na graduação.

De acordo com Mocelin (2009), de modo geral, o aumento acelerado da instrução educacional nas sociedades, com o crescimento no número de pesquisadores, pode ocorrer em razão não apenas da importância do conhecimento, mas também da valorização econômica da ciência e da tecnologia. $\mathrm{O}$ autor aponta ainda outros fatores que têm promovido a expansão da produção científica, como alterações na carreira docente, a ampliação dos concursos e os critérios de avaliação de projetos e de distribuição de recursos.

Uma forma de se perceber o desenvolvimento científico da área é por meio da análise da produção intelectual gerada pelos docentes e discentes envolvidos com os programas de pós-graduação stricto sensu, em termos quantitativos e qualitativos. Nesse sentido, Godoi e Xavier (2012) ressaltam que, em virtude da pressão por elevados índices de produtividade acadêmica, os esforços acabaram sendo deslocados da pesquisa e produção de conhecimento para a produção e publicação de artigos. Corroborando com esses autores, Bertero, Caldas e Wood (1999) afirmam que a pesquisa em administração de empresas tem crescido quantitativamente nos últimos anos, no entanto, parece evidente que a qualidade não tem acompanhado a quantidade.

Do exposto, o presente estudo é pautado no seguinte problema de pesquisa: Quais os principais aspectos associados à produção intelectual dos programas de pós-graduação stricto sensu brasileiros da Área de Administração Pública e de Empresas, Ciências Contábeis e Turismo? Dessa forma, tem-se como objetivo analisar as características que influenciam a produção intelectual desses programas avaliados pela CAPES no quadriênio 2013-2016, visando indicar os aspectos que mais contribuem para o desenvolvimento científico dessa área de conhecimento. Sua contribuição é, portanto, apresentar o atual panorama da produção intelectual dos programas de pós-graduação, bem como levantar informações que possam fundamentar discussões relacionadas ao desempenho dos programas de pós-graduação na geração e disseminação do conhecimento científico.

\section{REFERENCIAL TEÓRICO}

Apresentam-se nessa seção os principais temas que fundamentam o desenvolvimento do trabalho. São discutidos aspectos da avaliação da pós-graduação, bem como características da produção intelectual em Administração no Brasil.

\subsection{AVALIAÇÃO DA PÓS-GRADUAÇÃO STRICTO SENSU}

A avaliação dos programas de pós-graduação stricto sensu no Brasil é realizada pela CAPES desde 1976. Esse processo de avaliação é desempenhado por comissões de consultores do mais alto nível, vinculados às instituições das diferentes regiões do país. Essas comissões, denominadas Comissões de Avaliação, recebem orientações do 


\section{ASPECTOS ASSOCIADOS À PRODUÇÃO INTELECTUAL DOS \\ PROGRAMAS DE PÓS-GRADUAÇÃO EM ADMINISTRAÇÃO PÚBLICA E DE EMPRESAS, CIÊNCIAS CONTÁBEIS E TURISMO}

Conselho Técnico-Científico da Educação Superior (CTC-ES), no intuito de garantir uniformidade e padronização do processo de avaliação.

A avaliação dos programas de pós-graduação, que constitui o Sistema Nacional de Pós-Graduação (SNPG), atualmente acontece a cada quatro anos. O processo de avaliação para os programas de pós-graduação acontece em cinco etapas. A primeira delas consiste na disponibilização anual de informações por parte das instituições que oferecem cursos de pós-graduação stricto sensu, acerca das atividades desempenhadas no período avaliado, dados que são inseridos na Plataforma Sucupira, que faz parte da CAPES. Na segunda etapa, as informações são consolidadas pelos técnicos da CAPES e levadas à próxima fase, em que os programas são analisados pela comissão específica da área, e são atribuídas as notas aos programas (CAPES, 2016).

$\mathrm{Na}$ quarta fase os relatórios são examinados pelo CTC-ES, que apresenta uma conclusão sobre as notas de cada programa, dando sequência à quinta e última etapa, em que os pareceres do CTC-ES são enviados ao Conselho Nacional de Educação (CNE/MEC), resultando na aprovação e renovação de reconhecimento dos cursos (CAPES, 2016).

A atribuição de notas aos programas resulta da avaliação, pelas Comissões de Área, dos programas de pós-graduação, sendo concedidos os conceitos de 1 a 5 . Os programas conceituados com nota 5 são reavaliados e poderão receber as notas 6 ou 7 , sendo os critérios para se atribuir esses conceitos pautados na liderança em âmbito nacional e, também na inserção internacional de cada programa de pós-graduação (CAPES, 2016).

$\mathrm{Na}$ avaliação do quadriênio 2013-2016, as fichas de avaliação propostas contemplam critérios a serem analisados considerando-se três tipos de programas de pósgraduação, sendo os programas acadêmicos, os profissionais, e os programas profissionais em rede nacional.

A ficha de avaliação dos programas de mestrado e doutorado Acadêmico, tal como a ficha de avaliação dos mestrados profissionais, apresentam os cinco quesitos principais em seu corpo de avaliação, diferenciando-se em pesos: a) Proposta do Programa; b) Corpo Docente; c) Corpo Discente, Teses e Dissertações; d) Produção Intelectual e; e) Inserção Social.

Por sua vez, a ficha de avaliação dos programas de pós-graduação que oferecem mestrados profissionais em rede nacional apresenta um corpo de análise diferente, tendo apenas quatro quesitos: a) Avaliação da Rede e suas Associadas; b) Discentes e Egressos; c) Corpo Docente; e d) Inserção Social.

Considerando a avaliação dos programas de pós-graduação acadêmicos da área de Administração Pública e de Empresas, Ciências Contábeis e Turismo, pode-se notar que as variáveis com maior peso no processo são as que se referem ao Corpo Discente, Teses e Dissertações, assim como a Produção Intelectual, que têm, ambas, um peso de $35 \%$, totalizando $70 \%$ do processo de avaliação (CAPES, 2016). O quesito menos relevante para a análise é a Inserção Social, que pesa apenas 10\% no todo (CAPES, 2016). Considerando o quesito Corpo Discente pode-se destacar o subitem que se refere à qualidade das teses e dissertações, que por sua vez é analisado apenas para mestrados com mais de dois anos de funcionamento ou doutorados com mais de quatro anos. Além disso, são consideradas as produções científicas dos discentes e egressos dos programas de pós-graduação. Tal fato deve-se provavelmente à importância da consistência do programa por um período mínimo de anos.

$\mathrm{Na}$ avaliação dos mestrados profissionais, considerando aqueles avaliados pela comissão da área de Administração Pública e de Empresas, Ciências Contábeis e 


\section{ASPECTOS ASSOCIADOS À PRODUÇÃO INTELECTUAL DOS \\ PROGRAMAS DE PÓS-GRADUAÇÃO EM ADMINISTRAÇÃO PÚBLICA E \\ DE EMPRESAS, CIÊNCIAS CONTÁBEIS E TURISMO}

Turismo, podem-se destacar os itens Corpo Discente e Produção Intelectual, em que cada um deles tem peso de $30 \%$ da avaliação, totalizando $60 \%$ da composição da nota do programa (CAPES, 2016). Além disso, Corpo Docente e Inserção Social têm peso atribuído de $20 \%$ cada (CAPES, 2016). Considerando o item Corpo Discente, destaca-se o subitem da qualidade dos trabalhos de conclusão produzidos por discentes e egressos, sendo considerados para a análise os programas com dois ou mais anos de funcionamento, e que tenham artigos em periódicos ou livros, produção científica e produção tecnológica classificadas pela área. Em relação à Produção Intelectual, todos os elementos possuem atribuições equivalentes para a avaliação.

Vale ressaltar que o quesito Proposta do Programa consta como critério de análise nas fichas de avaliação, no entanto, esse aspecto não tem peso atribuído no diagnóstico dos programas acadêmicos ou profissionais, sendo realizadas apenas análises qualitativas acerca da proposta dos programas. Por sua vez, nos programas profissionais em rede nacional, este quesito não está no corpo de análise.

Por fim, na avaliação dos programas profissionais em rede nacional, nota-se que o quesito com maior peso atribuído é o de Discentes e Egressos, que tem valor de destaque na análise, sendo $40 \%$ do todo. Incorporado a esse quesito tem-se como mais relevante o subitem de qualidade dos trabalhos finais, que por sua vez mostra a adequação dos trabalhos à proposta do curso e sua divulgação. Esse quesito tem por base analisar os trabalhos de conclusão de curso e o número de egressos, fazendo uma proporção dentro de período de avaliação. Os outros quesitos têm peso igual na avaliação, todos com $20 \%$ (CAPES, 2016).

\subsection{AVALIAÇÃO DA PRODUÇÃO INTELECTUAL EM ADMINISTRAÇÃO PÚBLICA E DE EMPRESAS, CIÊNCIAS CONTÁBEIS E TURISMO}

Na avaliação da produção intelectual dos programas de pós-graduação considerase o quantitativo de artigos publicados em periódicos nacionais e internacionais, sendo que aqueles trabalhos publicados em periódicos considerados de maior relevância acadêmica proporcionam maior pontuação para o programa de pós-graduação. A classificação dos periódicos com produções de docentes, discentes e egressos nos cursos de Administração Pública e de Empresas, Ciências Contábeis e Turismo foi realizada pelo Comitê da Área, que por sua vez considerou somente periódicos que tiveram pelo menos uma publicação na área de avaliação no período de 2013 a 2016 (CAPES, 2017).

Os periódicos são classificados em estratos, denominados A1, A2, B1, B2, B3, B4, B5 e C. Para alcançar determinada classificação, os periódicos devem atender às condições estabelecidas pela comissão, cujos critérios incluem considerações sobre o impacto científico dos periódicos, mensurados por meio de índices que avaliam o número de citações dos artigos veiculados nos periódicos. Na avaliação do quadriênio 2013-2016, para alcançar o estrato A1, o periódico deve apresentar fator de impacto JCR superior a $1,4(67 \%)$ ou H-Scopus maior que $24(75 \%)$. No estrato A2, o fator de impacto JCR deve ser menor ou igual a 1,4 e maior que $0,7(33 \%)$, ou H-Scopus menor ou igual a 24 e maior que $9(50 \%)$ (CAPES, 2017).

Para obter classificação B1 o periódico deve estar na Scielo ou obter determinados valores em outras bases, como JCR menor ou igual a 0,7 , ou H-Scopus menor ou igual a 9, ou Índice Spell maior que 0,225 (67\%). O estrato B2 tem por condições estar indexado na base Redalyc, ser feito por editoras da área, estar na Scielo ou Índice Spell menor ou igual a 0,225 e maior que $0,114(33 \%)$. As classificações dos demais estratos são 


\section{ASPECTOS ASSOCIADOS À PRODUÇÃO INTELECTUAL DOS \\ PROGRAMAS DE PÓS-GRADUAÇÃO EM ADMINISTRAÇÃO PÚBLICA E \\ DE EMPRESAS, CIÊNCIAS CONTÁBEIS E TURISMO}

estabelecidas considerando aspectos como índice de atraso, tempo de existência do periódico e existência de algum dos indexadores definidos pela área (CAPES, 2017).

Os periódicos identificados como técnicos ou estritamente aplicados são atribuídos ao estrato $\mathrm{C}$ e usados para dar valor às produções tecnológicas na área de cada programa de pós-graduação. Alguns periódicos com destaque em sua produção em âmbito nacional tiveram suas classificações situadas um estrato acima, como por exemplo, se foi classificado em B1, passou-se para A2 (CAPES, 2017).

$\mathrm{Na}$ avaliação da produção intelectual dos programas de pós-graduação, são considerados dois indicadores, sendo i) pontuação média da produção de todos os docentes permanentes vinculados ao programa, e ii) pontuação considerando apenas as três melhores publicações dos docentes. No cômputo desses indicadores, são considerados os artigos publicados por professores, discentes e egressos e lhes são atribuídos pontos de acordo com cada estrato como segue: A1 (100 pontos), A2 (80 pontos), B1 (60 pontos), B2 (50 pontos), B3 (30 pontos), B4 (20 pontos), B5 (10 pontos) e C (0 pontos) (CAPES, 2017).

\subsection{PRODUÇÃO INTELECTUAL EM ADMINISTRAÇÃO NO BRASIL}

As pesquisas sobre a produção científica da área de Administração no Brasil começaram a ser desenvolvidas na década de 1990, podendo-se citar os estudos de Siqueira (1988) e Machado-da-Silva, Cunha e Amboni (1990). Posteriormente, uma lista de pesquisas com essa temática inclui os trabalhos de Bertero e Keinert (1994), Hoppen (1998), Bertero, Caldas e Wood (1999, 2005), Vergara e Pinto (2001), Kuenzer e Moraes (2005), Wood e Chueke (2008), Leite (2008), Alcadipani (2011), Wood e Costa (2014) e Cunha, Defina e Passador (2014).

O campo da produção científica em administração no Brasil apresenta uma trivialidade quanto aos estudos teóricos produzidos, em que esses ensaios são realizados pelos pesquisadores sem haver preocupação com a sua qualidade e com temas relacionados à aplicação prática (Machado-da-Silva et al., 1990). Nesse aspecto, Cunha et al. (2014) concluem que é necessário um esforço para identificar se os estudos apresentados com qualidade têm origem em ensaios teóricos ou empíricos.

Kuenzer e Moraes (2005) também corroboram com essa ideia e alegam que a intensa produção é um fator negativo, pois a necessidade por traçar metas quantitativas atrapalha a qualidade das produções científicas, ou seja, a avaliação quantitativa se destaca em relação à qualitativa.

Confirmando essa concepção, Bertero et al. (1999, 2005) afirmam que as produções científicas não obtiveram crescimento satisfatório na sua qualidade ao longo do tempo, de modo a evidenciar vulnerabilidades metodológicas e teóricas. Alcadipani (2011) reafirma essa percepção, salientando que as publicações são complexas e que necessitam de tempo para o seu desenvolvimento.

Ao analisar o estado da arte da área de Sistemas de Informação em revistas científicas de Administração, Hoppen (1998) constatou que, de modo geral, os artigos baseados em pesquisa empírica apresentaram qualidade científica média-baixa, como consequência do pouco rigor no que se refere ao desenho de pesquisa e à validade dos instrumentos. $\mathrm{O}$ autor apontou ainda falta de cuidado com relação ao rigor científico no desenvolvimento das pesquisas e falta de informações importantes na apresentação dos resultados nos artigos.

Por sua vez, Bertero et al. (1999, p. 148) descrevem a produção científica em administração de empresas como "periférica, epistemologicamente falha, 


\section{ASPECTOS ASSOCIADOS À PRODUÇÃO INTELECTUAL DOS \\ PROGRAMAS DE PÓS-GRADUAÇÃO EM ADMINISTRAÇÃO PÚBLICA E DE EMPRESAS, CIÊNCIAS CONTÁBEIS E TURISMO}

metodologicamente deficiente, sem originalidade e prática, em grande escala, mimetismo mal-informado".

Nesse contexto de abrangência e publicidade das pesquisas da área, Cunha et al. (2014) apontam que uma produção acadêmica escrita em um idioma estrangeiro retrata uma qualidade no conteúdo dos artigos, e que trabalhos de qualidade estão sendo elaborados com foco em uma possível publicação internacional. No entanto, para Wood e Costa (2014) existem obstáculos com as diferentes agendas de pesquisa e com a própria língua inglesa, que impedem os pesquisadores brasileiros quanto às possíveis publicações estrangeiras em revistas de maior abrangência e de alto impacto.

Com relação ao referencial teórico utilizado nas pesquisas em administração no Brasil, de acordo com Vergara e Pinto (2001), nota-se que as referências americanas têm sido as mais utilizadas, corroborando com os achados de Bertero e Keinert (1994). No referido estudo, Bertero e Keinert (1994) relatam ainda que os assuntos abordados nas pesquisas também indicam que os estudos brasileiros seguem a tendência do momento nos países onde as ideias se originam, acarretando a presença dos diversos modismos que se sucedem na produção da área.

Considerando o importante papel desempenhado pela pós-graduação na produção do conhecimento científico (Leite, 2008), sendo a geração e a veiculação da produção científica atividades essenciais dos programas de pós-graduação (Wood \& Chueke, 2008), a recente ampliação da pós-graduação da área de Administração Pública e de Empresas, Ciências Contábeis e Turismo poderia representar melhorias na produção intelectual da área. No entanto, segundo Bertero et al. (1999), o sistema de pós-graduação no Brasil é grande, mas não possui qualidade mundial, e apesar da pesquisa em administração ter crescido quantitativamente nos últimos anos, a qualidade parece não ter acompanhado a quantidade.

Em estudo realizado por Moreira, Cunha, Ferreira e Silveira (2009), verificou-se que os programas de pós-graduação acadêmicos da área de Administração, Ciências Contábeis e Turismo vinculados às instituições privadas de ensino apresentaram desempenho superior em termos de produção científica e titulação de mestres e doutores, se comparados àqueles programas da rede pública. $\mathrm{O}$ estudo ainda mostrou que o desempenho dos programas de pós-graduação vinculados às instituições públicas de ensino superior mostrou-se mais heterogêneo.

Bertero et al. (1999) defendem que o fortalecimento do campo da administração no Brasil depende do estabelecimento de critérios de avaliação mais claros e bem definidos e que, em resposta ao crescimento quantitativo das pesquisas na área, é necessário se voltar para discussões acerca da qualidade da produção científica.

\section{PROCEDIMENTOS METODOLÓGICOS}

A pesquisa é caracterizada como quantitativa, explicativa e ex post facto. Quantitativa por utilizar de ferramental estatístico para analisar dados numéricos relativos à produção intelectual dos programas de pós-graduação. Explicativa, por ter como principal objetivo identificar os fatores que contribuem para a ocorrência de determinado fenômeno. E ex post facto por se tratar da análise de variáveis que representam a produção intelectual passada dos programas de pós-graduação, não sendo possível a interferência do pesquisador sobre essas variáveis.

Buscando garantir uniformidade ao estudo, uma vez que os critérios utilizados pela CAPES na avaliação dos programas de pós-graduação são revistos e ajustados a cada quatro anos, optou-se em trabalhar com os programas de pós-graduação stricto sensu em 


\section{ASPECTOS ASSOCIADOS À PRODUÇÃO INTELECTUAL DOS \\ PROGRAMAS DE PÓS-GRADUAÇÃO EM ADMINISTRAÇÃO PÚBLICA E DE EMPRESAS, CIÊNCIAS CONTÁBEIS E TURISMO}

Administração Pública e de Empresas, Ciências Contábeis e Turismo avaliados pela CAPES no quadriênio 2013-2016.

Portanto, a população da pesquisa foi representada por 182 programas de pósgraduação avaliados pela comissão de avaliação da área de Administração, Ciências Contábeis e Turismo, sendo os dados coletados em planilha de indicadores disponibilizada no portal da CAPES, na qual consta a produção intelectual distribuída segundo a estratificação Qualis, bem como outras informações relativas aos programas de pós-graduação avaliados no quadriênio 2013-2016.

O desenvolvimento do trabalho ocorreu por meio dos seguintes procedimentos de investigação: (i) cálculo da pontuação média por docente da produção intelectual dos programas de pós-graduação stricto sensu em Administração Pública e de Empresas, Ciências Contábeis e Turismo; e (ii) identificação dos fatores associados à produção intelectual dos programas de pós-graduação.

O cálculo da pontuação da produção intelectual dos programas de pós-graduação teve por objetivo criar uma variável que represente o nível da produção intelectual dos programas. O cômputo dessa variável considerou a soma do número de artigos publicados em periódicos referenciados no Qualis, de 2013 a 2016, ponderados pelos pesos relativos a cada estrato, conforme os critérios utilizados pela comissão de avaliação da área de Administração Pública e de Empresas, Ciências Contábeis e Turismo na avaliação da produção intelectual dos programas de pós-graduação (CAPES, 2016). Os pesos dos artigos nos estratos A1, A2, B1, B2, B3, B4 e B5 são, respectivamente, 100, 80, 60, 50, 30,20 e 10.

Ao atribuir maior peso àqueles artigos publicados em periódicos dos estratos mais relevantes, acredita-se estar incluindo aspectos qualitativos na medida de produção intelectual dos programas de pós-graduação, tendo em vista que tais periódicos alcançam maior impacto na comunidade científica.

Para analisar a produtividade dos programas, foi calculada a produção média por docente permanente. A Equação 1 demonstra o cálculo da variável produção intelectual média por docente (ProDoc):

$$
\text { ProDoc }=\frac{(A 1 \times 100)+(A 2 \times 80)+(B 1 \times 60)+(B 2 \times 50)+(B 3 \times 30)+(B 4 \times 20)+(B 5 \times 10)}{\text { qtde média anual de Docentes Permanentes }}
$$

A análise dos fatores associados à produção intelectual dos programas de pósgraduação se deu por meio da análise de regressão múltipla pelo método dos mínimos quadrados ordinários (MQO), visando estimar e validar os parâmetros do modelo.

A análise de regressão múltipla é uma técnica estatística usada para analisar a relação entre uma única variável dependente e várias variáveis independentes ou preditoras (Hair, Black, Babin, Anderson, \& Tatham, 2009). O objetivo da análise de regressão múltipla é usar as variáveis independentes, cujos valores são conhecidos, para prever os valores da variável dependente. Cada variável independente é ponderada pelo procedimento de regressão para garantir máxima previsão a partir do conjunto de variáveis independentes. Dessa forma, quanto mais significativo for o peso de uma variável isolada ou de um conjunto de variáveis explicativas, tanto mais se poderá afirmar que alguns fatores afetam mais o comportamento de uma variável de resposta especificamente procurada do que outros (Gujarati, 2005; Hair et al., 2009; Wooldridge, 2006).

O formato geral da equação de regressão linear múltipla (Equação 2), de acordo com Triola (2005), é: 


\section{ASPECTOS ASSOCIADOS À PRODUÇÃO INTELECTUAL DOS \\ PROGRAMAS DE PÓS-GRADUAÇÃO EM ADMINISTRAÇÃO PÚBLICA E \\ DE EMPRESAS, CIÊNCIAS CONTÁBEIS E TURISMO}

$$
\hat{y}=b_{0}+b_{1} x_{1}+b_{2} x_{2}+\cdots+b_{k} x_{k}
$$

em que $\hat{y}$ é o valor predito da variável dependente; $b_{0}$ é a estimativa do intercepto (ou valor de y quando os regressores são zero); $b_{1}, b_{2}, \ldots, b_{k}$ são estimativas dos coeficientes das variáveis independentes; e $x_{1}, x_{2}, \ldots, x_{k}$ são as variáveis independentes.

No presente estudo, a variável dependente considerada é a produção intelectual média por docente permanente (ProDoc) de cada programa de pós-graduação e as variáveis independentes constam de características dos programas cuja relação com a produção intelectual se pretende analisar, conforme segue:

i) Tempo de Funcionamento (TemFun): representa o número de anos que o programa de pós-graduação esteve em atividade até o ano de 2016, último ano considerado na avaliação do quadriênio;

ii) Categoria Administrativa (CatAdm): variável dummy que representa o vínculo do programa de pós-graduação a instituição privada (0) ou pública (1) de ensino;

iii) Total de Docentes e Discentes (DocDis): média anual da soma do número docentes permanentes e discentes, de 2013 a 2016, empregada como proxy do tamanho dos programas;

iv) Artigos em Anais de Eventos (ArtAna): número de artigos completos publicados em anais de eventos científicos, com autoria ou coautoria de docentes vinculados ao programa de pós-graduação;

v) Bolsas por Discentes (BolDis): percentual médio de bolsas recebidas pelos discentes dos programas de pós-graduação, considerando as bolsas recebidas no quadriênio 2013-2016.

Ressalta-se que devido a problemas de heterocedasticidade detectados previamente, para a análise de regressão foi realizada a correção robusta de White. Esse método de correção impede que o modelo incorra em problemas causados pela violação da hipótese do MQO, o qual defende que as variâncias dos termos de erro devem ser constantes (Hair et al., 2009). Foram ainda testadas as hipóteses de multicolinearidade e normalidade dos resíduos, as quais foram atendidas.

A seguir são apresentados os resultados do modelo proposto, bem como a discussão sobre os fatores associados à produção intelectual dos programas de pósgraduação stricto sensu em Administração Pública e de Empresas, Ciências Contábeis e Turismo no quadriênio 2013-2016.

\section{RESULTADOS E DISCUSSÕES}

Analisando a produção intelectual dos programas de pós-graduação stricto sensu brasileiros da área de Administração Pública e de Empresas, Ciências Contábeis e Turismo, nota-se que a maior concentração de artigos ocorre em periódicos do estrato Qualis B2 (Tabela 1).

Tabela 1 - Estatísticas descritivas da produção intelectual dos programas de pósgraduação stricto sensu em Administração Pública e de Empresas, Ciências Contábeis e Turismo, 2013-2016

\begin{tabular}{ccccccccc}
\hline & A1 & A2 & B1 & B2 & B3 & B4 & B5 & C \\
\hline Média & \multirow{2}{*}{6,2363} & 25,2692 & 28,7308 & 31,3956 & $\begin{array}{c}29,972 \\
5\end{array}$ & 28,4670 & 5,8571 & 1,4066 \\
& & & & & 5 & & & \\
\hline
\end{tabular}




\section{ASPECTOS ASSOCIADOS À PRODUÇÃO INTELECTUAL DOS PROGRAMAS DE PÓS-GRADUAÇÃO EM ADMINISTRAÇÃO PÚBLICA E DE EMPRESAS, CIÊNCIAS CONTÁBEIS E TURISMO}

\begin{tabular}{lcccccccc}
\hline Desv. & 10,7843 & 27,5278 & 29,8816 & 32,0482 & 29,229 & 29,1854 & 7,2044 & 2,0108 \\
Padrão & & & & & 0 & & & \\
Coef. & 172,93 & 108,94 & 104,01 & 102,08 & & 102,52 & 123,00 & 142,96 \\
Variação & $\%$ & $\%$ & $\%$ & $\%$ & $97,52 \%$ & $\%$ & $\%$ & $\%$ \\
Máximo & 63 & 168 & 172 & 228 & 179 & 230 & 39 & 10 \\
Mínimo & 0 & 0 & 0 & 0 & 0 & 0 & 0 & 0 \\
TOTAL & 1.135 & 4.599 & 5.229 & 5.714 & 5.455 & 5.181 & 1.066 & 256 \\
$\begin{array}{l}\text { Frequênci } \\
\text { a (\%) }\end{array}$ & $3,96 \%$ & $16,06 \%$ & $18,26 \%$ & $19,95 \%$ & $19,05 \%$ & $18,09 \%$ & $3,72 \%$ & $0,89 \%$ \\
\hline
\end{tabular}

Fonte: Resultados da pesquisa (2020).

Entretanto, é possível verificar a ocorrência de volume de publicação semelhante em periódicos dos estratos B1, B3 e B4, o que indica existir maior concentração no número de publicações em periódicos de estratos medianos.

Vale destacar a expressiva variação dos dados em torno da média em todos os tipos de publicações, denotando existir grande heterogeneidade em relação à produção intelectual dos programas de pós-graduação analisados, principalmente ao considerar os níveis de publicação em periódicos do estrato $\mathrm{A} 1$, tendo em vista que o desvio relativo à média desse estrato atinge mais de $172 \%$ (Tabela 1).

Os dados na Tabela 2 referem-se às principais estatísticas descritivas da variável que representa a produção intelectual média por docente permanente (ProDoc), bem como as demais variáveis consideradas na análise de regressão múltipla, quais sejam: i) tempo de funcionamento do programa (TemFun); ii) categoria administrativa (CatAdm); iii) total de docentes e discentes (DocDis); iv) artigos em anais de eventos (ArtAna); e v) número de bolsas recebidas pelos discentes (BolDis) no quadriênio 2013-2016.

Tabela 2 - Estatísticas descritivas das variáveis utilizadas na análise de regressão múltipla

\begin{tabular}{lcccccc}
\hline & ProDoc & TemFun & CatAdm & DocDis & ArtAna & BolDis \\
\hline Média & 462,8574 & 10,4725 & 0,6538 & 84,7514 & 175,6978 & $6,0552 \%$ \\
Desv. Padrão & 300,7466 & 11,5033 & 0,4757 & 58,8789 & 174,6908 & $8,0818 \%$ \\
Coef. Variação & $64,98 \%$ & $109,84 \%$ & $72,76 \%$ & $69,47 \%$ & $99,43 \%$ & $133,47 \%$ \\
Máximo & $1.837,50$ & 49,00 & 1,00 & 449,67 & $1.140,00$ & $51,0526 \%$ \\
Mínimo & 10,77 & 0,00 & 0,00 & 18,00 & 2,00 & $0,0000 \%$ \\
\hline
\end{tabular}

Fonte: Resultados da pesquisa (2020).

A produção intelectual média por docente permanente (ProDoc) mostra a pontuação média por docente alcançada pelos programas. Essa pontuação pondera a quantidade de artigos publicados e o estrato Qualis do periódico em que os artigos foram publicados. Essa variável apresentou variação de aproximadamente $65 \%$ em torno da média, transparecendo que há uma heterogeneidade muito grande na produção nesses programas. O programa que mais publicou, na média por docente permanente, alcançou uma média de $1.837,5$ pontos, enquanto o programa com a menor produção média no quadriênio 2013-2016 obteve cerca de 10 pontos. 


\section{ASPECTOS ASSOCIADOS À PRODUÇÃO INTELECTUAL DOS \\ PROGRAMAS DE PÓS-GRADUAÇÃO EM ADMINISTRAÇÃO PÚBLICA E DE EMPRESAS, CIÊNCIAS CONTÁBEIS E TURISMO}

A variável tempo de funcionamento (TemFun) reflete o nível de consolidação do programa. Assim sendo, busca-se verificar se o tempo de existência do programa é capaz de influenciar sua capacidade para gerar conhecimento científico, bem como proporcionar melhores condições para o funcionamento das atividades de pós-graduação, expandindo sua produção intelectual.

Nesse sentido, os programas de pós-graduação stricto sensu em Administração Pública e de Empresas, Ciências Contábeis e Turismo no quadriênio 2013-2016 possuem média de 10 anos de funcionamento, apresentando programas com até 49 anos em atividade, e por outro lado outros que começaram seus trabalhos no período analisado. Em vista disso expõe-se que há uma grande heterogeneidade no tempo de funcionamento desses programas.

A variável CatAdm expressa o vínculo dos programas, seja com instituições privadas ou públicas. A variável foi categorizada de forma que os programas vinculados às instituições privadas foram representados por (0) e aqueles pertencentes às instituições públicas por (1). Do total de 182 programas de pós-graduação, 119 deles estão vinculados às instituições públicas e 63 às instituições privadas.

Por conseguinte, a variável DocDis é a soma do número de docentes e discentes e expressa a dimensão dos programas de pós-graduação. A inclusão da referida variável no estudo visa analisar se o tamanho dos programas é capaz de influenciar na produção intelectual. Acredita-se que o maior número de professores possa facilitar o oferecimento de disciplinas, a orientação acadêmica e a manutenção das linhas de pesquisa, ampliando a capacidade para receber novos alunos. A quantidade de discentes alocados ao programa também pode contribuir com a produção dos mesmos, uma vez que esses influenciam na capacidade produtiva, tendo em vista que representam parte fundamental do desenvolvimento de pesquisas. Analisando essa variável, nota-se que, em média, os programas de pós-graduação são compostos por 84 docentes e discentes, anualmente. Ressalta-se que essa variável se apresenta muito dispersa, tendo em vista existirem programas com até 449 professores e alunos, ao mesmo tempo em que alguns contam com o quantitativo bem reduzido de pessoal, como aponta o valor mínimo de 18 docentes e discentes por ano.

Considerando a publicação de artigos em anais de eventos (ArtAna) nota-se novamente desproporção entre os programas analisados, tendo em vista as diferenças entre os valores de máximo e mínimo. A Tabela 2 indica programas com 1.140 publicações no período, enquanto outros computaram apenas 2 publicações. Espera-se que, quanto maior a participação dos docentes e discentes em eventos científicos, com a divulgação dos resultados de suas pesquisas em anais desses eventos, melhores serão as condições para que os programas de pós-graduação possam publicar os artigos em periódicos conceituados, ampliando o potencial de contribuição junto à área de conhecimento.

A divulgação de artigos em eventos científicos se mostra de suma importância no sentido da troca de experiências entre os acadêmicos da área, entretanto, essas publicações são consideradas provisórias. Logo, espera-se que sobrevenha um amadurecimento dessas publicações, e que as mesmas sejam sujeitas a publicação em periódicos posteriormente.

Por fim, a variável BolDis expressa o percentual médio de discentes de mestrado e doutorado atendidos por bolsas em cada programa de pós-graduação. A média geral indica que aproximadamente $6 \%$ dos alunos de pós-graduação stricto sensu receberam bolsas no quadriênio 2013-2016. Observa-se, no entanto, uma variação em torno da média de $133 \%$, em que o valor mínimo é representado por programas que não concederam 


\section{ASPECTOS ASSOCIADOS À PRODUÇÃO INTELECTUAL DOS \\ PROGRAMAS DE PÓS-GRADUAÇÃO EM ADMINISTRAÇÃO PÚBLICA E \\ DE EMPRESAS, CIÊNCIAS CONTÁBEIS E TURISMO}

bolsas e outros que ofereceram, em média, bolsas a cerca de $51 \%$ dos discentes matriculados.

O oferecimento de bolsas de estudos busca proporcionar condições para a maior dedicação do discente no desenvolvimento de suas atividades acadêmicas. Em vista disso, procura-se uma relação positiva entre a variável BolDis e a produção intelectual por docente permanente dos programas de pós-graduação (ProDoc).

Os resultados apresentados na Tabela 3 indicam que todas as variáveis se mostram estatisticamente significativas na determinação do nível de produção intelectual média por docente permanente (ProDoc) dos programas de pós-graduação stricto sensu em Administração Pública e de Empresas, Ciências Contábeis e Turismo no quadriênio 20132016.

Tabela 3 - Variáveis associadas à produção intelectual dos programas de pós-graduação stricto sensu em Administração Pública e de Empresas, Ciências Contábeis e Turismo, 2013-2016

\begin{tabular}{lrrrlrr}
\hline Variável & Coeficiente & Erro padrão Robusto & \multicolumn{1}{c}{$\mathbf{T}$} & \multicolumn{2}{c}{$\mathbf{P}>|\mathbf{t}|$} & [95\% Intervalo Confiança] \\
\hline TemFun & 6,3545 & 2,0923 & 3,04 & $0,003^{*}$ & 2,2252 & 10,4838 \\
CatAdm & $-117,2952$ & 28,9870 & $-4,05$ & $0,000^{*}$ & $-174,5019$ & $-60,0884$ \\
DocDis & $-1,4772$ & 0,5115 & $-2,89$ & $0,004^{*}$ & $-2,4867$ & $-0,4677$ \\
ArtAna & 1,3039 & 0,1391 & 9,37 & $0,000^{*}$ & 1,0294 & 1,5785 \\
BolDis & 5,7790 & 1,6672 & 3,47 & $0,001^{*}$ & 2,4887 & 9,0694 \\
_cons & 334,1054 & 45,5611 & 7,33 & $0,000^{*}$ & 244,1889 & 424,0219 \\
& & & & & & \\
$\mathbf{R}^{2}$ & 0,6938 & & & & & \\
Teste F & 47,83 & & & $0,000^{*}$ & &
\end{tabular}

Fonte: Resultados da pesquisa (2020).

* significativo a $1 \%$

Verificou-se que o teste $\mathrm{F}$ apresentou coeficiente significativo a $1 \%$, rejeitando a hipótese nula de que todos os coeficientes de regressão sejam iguais a zero. Conjuntamente, nota-se que o valor do R-quadrado sugere que $69 \%$ das variações percebidas na produção intelectual por docente (ProDoc) podem ser explicadas pelas variáveis incluídas no estudo, indicando um bom ajustamento do modelo.

Apesar de todas as variáveis se mostrarem significativas em relação à produção média por docente permanente (ProDoc), nem todas apresentaram relação positiva com ela. Partindo desse fato, percebe-se que a variável tempo de funcionamento (TemFun) tem relação positiva com a variável dependente. Isso pressupõe que quanto maior o tempo de atuação de um programa, maior será a produção intelectual média dos docentes permanentes, provavelmente em função da maior experiência e melhor alinhamento das práticas de pesquisa da equipe de professores.

A variável CatAdm tem por finalidade identificar se a categoria administrativa da instituição que está vinculado o programa de pós-graduação, se privada ou pública, 


\section{ASPECTOS ASSOCIADOS À PRODUÇÃO INTELECTUAL DOS \\ PROGRAMAS DE PÓS-GRADUAÇÃO EM ADMINISTRAÇÃO PÚBLICA E DE EMPRESAS, CIÊNCIAS CONTÁBEIS E TURISMO}

influencia na produção científica. A análise de regressão mostrou que a categoria administrativa é um aspecto capaz de influenciar a produção intelectual da área de Administração Pública e de Empresas, Ciências Contábeis e Turismo. De acordo com a Tabela 3, nota-se um coeficiente com valor negativo, assim sugerindo que os programas das instituições privadas são mais produtivos que aqueles das instituições públicas. Esse resultado corrobora como os achados da pesquisa desenvolvida por Moreira et al. (2009), em que os programas de pós-graduação acadêmicos da área de Administração, Ciências Contábeis e Turismo oferecidos por instituições privadas mostraram desempenho superior, em relação à publicação científica e titulação de mestres e doutores nos anos de 2004 a 2006, que aqueles programas vinculados às instituições públicas de ensino.

Analisando a influência do tamanho dos programas de pós-graduação, aqui representado pela média anual do quantitativo de docentes e discentes matriculados, (DocDis), notou-se uma relação negativa com a variável dependente. Esse resultado demonstra que a quantidade de professores e alunos de um programa não reflete necessariamente que a produção de artigos será maior, tendo em vista que os maiores programas de pós-graduação se mostraram associados às menores médias de produção intelectual. Do exposto, conclui-se que a produção científica talvez dependa muito mais de fatores que não apenas quantitativos, mas, por outro lado, esteja sujeita a estímulos capazes de motivar a produção intelectual, tornando os pesquisadores mais engajados e comprometidos a realizar pesquisas de qualidade.

Foi possível verificar ainda que a publicação de artigos em anais de eventos (ArtAna) apresentou relação positiva com a produção intelectual média por docente (ProDoc). Isso demonstra que quanto maior a participação dos docentes e discentes dos programas de pós-graduação em eventos acadêmicos da área e com a publicação de resultados de pesquisas, maior será a produção científica em periódicos.

Como já mencionado, a publicação de artigos em anais de eventos é considerada uma produção provisória. Contudo, acredita-se que, havendo o compartilhamento de resultados das pesquisas com outros pesquisadores externos ao programa, pode-se promover o amadurecimento desses trabalhos com vistas a gerar publicações definitivas em periódicos. Desse modo, recomenda-se o incentivo à participação de docentes e discentes em eventos científicos, bem como a apresentação de artigos resultantes das pesquisas realizadas.

Por fim, verificou-se que o percentual médio de discentes contemplados com bolsas nos programas de pós-graduação (BolDis) influencia positivamente na produção intelectual por docente permanente (ProDoc). Essa constatação evidencia a importância do pagamento de bolsas aos alunos de mestrado e doutorado para que os mesmos possam se dedicar integralmente ao desenvolvimento de suas atividades acadêmicas, incluindo a realização de pesquisas de qualidade que sejam capazes de produzir artigos relevantes e que contribuam com o desenvolvimento científico da área de conhecimento.

\section{CONSIDERAÇÕES FINAIS}

O objetivo geral do estudo foi desenvolver uma análise da produção intelectual dos programas de pós-graduação stricto sensu brasileiros da área de Administração Pública e de Empresas, Ciências Contábeis e Turismo, procurando identificar fatores que influenciaram a produção científica no período analisado.

Observou-se que houve uma maior concentração de artigos publicados em periódicos do estrato B2. Porém essa diferença não se mostra muito expressiva, já que estratos como B1, B3 e B4 apresentaram resultados muito próximos em relação ao estrato 


\section{ASPECTOS ASSOCIADOS À PRODUÇÃO INTELECTUAL DOS \\ PROGRAMAS DE PÓS-GRADUAÇÃO EM ADMINISTRAÇÃO PÚBLICA E DE EMPRESAS, CIÊNCIAS CONTÁBEIS E TURISMO}

líder em números. Vale destacar uma expressiva quantidade de artigos em periódicos do estrato A2, onde se encontram trabalhos com ampla influência nacional e alcance internacional.

Houve uma expressiva variação em torno da média em todos os estratos apresentados no estudo, podendo observar variações com até $172 \%$, como no estrato A1, e variação mínima de $97 \%$, cujo valor esteve presente nos periódicos de estrato B3. Esses dados demonstram existir uma grande heterogeneidade em relação aos veículos utilizados para a publicação científica dos programas analisados.

Ao considerar a produção intelectual média por docente permanente, nota-se também alto coeficiente de variação, tendo em vista que a pontuação média de determinados programas alcançou patamares bem distintos, em que alguns alcançaram 1.837 pontos, enquanto outros apenas 10 pontos. Tais fatores transparece haver também uma heterogeneidade muito grande em relação à produção intelectual dos programas de pós-graduação stricto sensu em Administração Pública e de Empresas, Ciências Contábeis e Turismo.

Vale destacar a grande variação no tempo de funcionamento dos programas de pós-graduação do estudo, apresentando alguns com quase meio século de atuação, e outros que começaram seus trabalhos no período analisado pela pesquisa. A variável nos mostra que quanto maior for o tempo de funcionamento, melhores serão as condições para o programa se estabelecer, seja gerando conhecimento científico, ou proporcionando condições melhores para o mesmo.

Por conseguinte, a variável Categoria Administrativa mostrou o impacto das instituições públicas e privadas na produção científica. Os dados retratam a maior quantidade de programas vinculados às instituições públicas com um total de 119 , contra 63 programas ligados à rede privada. Apesar disso, verificou-se que os programas vinculados às instituições privadas produziram e contribuíram mais para a produção científica no período analisado.

Considerando o tamanho dos programas, representado pela média anual de docentes e discentes, nota-se relação negativa com a produção científica, pressupondo que a quantidade de professores e alunos de um determinado programa, não necessariamente acarreta maior produção de trabalhos. Isso reforça a ideia de que se deve atentar não apenas a fatores quantitativos, mas também buscar incentivar e engajar o grupo de professores a alunos envolvidos.

Destacam-se também os dados apresentados em relação à publicação em anais de eventos, podendo observar uma grande discrepância no total de publicações obtidas por determinados programas comparados a outros. Essas informações em conjunto com a variável dependente concluíram que quanto maior a participação dos docentes e discentes em eventos acadêmicos com publicações, maior será a produção intelectual disseminada por meio de periódicos.

Por fim, o percentual médio de discentes que obtiveram bolsas nos programas de pós-graduação mostrou ter relação positiva com a produção científica, pressupondo que se deve haver esse investimento no pagamento de bolsas, pois o recebimento de bolsa torna-se um incentivo à dedicação integral para a produção das atividades acadêmicas.

Todas as variáveis mostraram-se significativas com a produção intelectual por docente permanente, no entanto, considerando os valores do coeficiente, nota-se que apenas três delas apresentaram relação positiva com a variável independente, quais sejam: tempo de funcionamento, artigos em anais de eventos e percentual médio de bolsas recebidas pelos discentes. Essa constatação sugere que a consolidação dos programas ao longo dos anos contribui para a maior capacidade dos docentes e discentes para gerar 


\section{ASPECTOS ASSOCIADOS À PRODUÇÃO INTELECTUAL DOS \\ PROGRAMAS DE PÓS-GRADUAÇÃO EM ADMINISTRAÇÃO PÚBLICA E \\ DE EMPRESAS, CIÊNCIAS CONTÁBEIS E TURISMO}

publicações em periódicos. Além disso, a participação dos docentes e discentes da pósgraduação em eventos científicos, com a respectiva apresentação de artigos, deve ser incentivada. A relação positiva entre o pagamento de bolsas aos discentes e a produção intelectual dos programas de pós-graduação stricto sensu em Administração Pública e de Empresas, Ciências Contábeis e Turismo, evidencia a importância das agências de apoio à pesquisa no desenvolvimento científico, além do auxílio no fomento ao desenvolvimento dos discentes de pós-graduação.

Como sugestão para futuras pesquisas, recomenda-se que a abordagem teóricometodológica aqui apresentada seja ampliada para outros períodos de tempo. Também, que sejam feitas pesquisas qualitativas para aprofundar o tema e conhecer melhor as características dos programas de pós-graduação stricto sensu e os problemas enfrentados, de forma a contribuir com o desenvolvimento científico dessa área de conhecimento.

\section{REFERÊNCIAS}

Alcadipani, R. (2011). Academia e fábrica de sardinhas. Organizações e Sociedade, 18(57), 345-348.

Bertero, C. O., Caldas, M. P., \& Wood, T., Jr. (1999). Produção científica em administração de empresas: provocações, insinuações e contribuições para um debate local. Revista de Administração Contemporânea, 3(1), 147-178.

Bertero, C. O., Caldas, M. P., \& Wood, T., Jr. (Coord.). (2005). Produção científica em administração no Brasil: o estado da arte (Cap. 1, pp. 1-17). São Paulo: Atlas.

Bertero, C. O., \& keinert, T. M. M. A evolução da análise organizacional no Brasil (196193). (1994). Revista de Administração de Empresas, 34(3), 81-90.

Coordenação de Aperfeiçoamento de Pessoal de Nível Superior. (2016). Documento de Área: Administração Pública e de Empresas, Ciências Contábeis e Turismo. Recuperado em 31 maio, 2021, de https://www.gov.br/capes/pt-br/centrais-deconteudo/27_ADMI_doc_area_2016_final_20jan2017.pdf

Coordenação de Aperfeiçoamento de Pessoal de Nível Superior. (2017). Relatório da Avaliação Quadrienal 2017: Administração Pública e de Empresas, Ciências Contábeis e Turismo. Recuperado em 31, maio, 2021, de https://www.gov.br/capes/pt-br/centraisde-conteudo/20122017-administracao-quadrienal-pdf

Coordenação de Aperfeiçoamento de Pessoal de Nível Superior. (2019). Documento de Área. Área 27: Administração Pública e de Empresas, Ciências Contábeis e Turismo. Recuperado em 31, maio, 2021, de https://www.gov.br/capes/pt-br/centrais-deconteudo/adm-pdf

Cunha, J. A. C. da, Defina, D. A., \& Passador, J. L. (2014). Qualidade da Produção Científica no Brasil: um enfoque nos trabalhos premiados pelos congressos da ANPAD 2004-2012. Revista de Ciências da Administração, 16(38),11-28.

Godoi, C.K., \& Xavier, W. G. (2012). O produtivismo e suas anomalias. Cad. EBAPE.BR, $10(2), 456-465$. 


\section{ASPECTOS ASSOCIADOS À PRODUÇÃO INTELECTUAL DOS \\ PROGRAMAS DE PÓS-GRADUAÇÃO EM ADMINISTRAÇÃO PÚBLICA E \\ DE EMPRESAS, CIÊNCIAS CONTÁBEIS E TURISMO}

Gujarati, D. (2005). Econometria básica (4a ed.). Rio de Janeiro: Elsevier.

Hair, J. F., Jr., Black, W. C., Babin, B. J., Anderson, R. E., \& Tatham, R. L. (2009). Análise Multivariada de Dados (6a ed.). Porto Alegre: Bookman.

Hoppen, N. (1998). Sistemas de informação no Brasil: uma análise dos artigos científicos dos anos 90. Revista de Administração Contemporânea, 2(3), 151-177.

Kuenzer, A., \& Moraes, M. (2005). Temas e tramas na pós-graduação em educação. Educação \& Sociedade, 26(93), 1.341-1.362.

Leite, G. A., Filho. (2008). Padrões de produtividade de autores em periódicos e congressos na área de Contabilidade no Brasil: um estudo bibliométrico. Revista de Administração Contemporânea, 12(2), 533-554.

Machado-da-Silva, C. L.; Cunha, V. C.; \& Amboni, N. (1990). Organizações: O estado da arte da produção acadêmica no Brasil. Anais do Encontro Nacional da Associação Nacional de Pós-Graduação e Pesquisa em Administração, Belo Horizonte, MG, Brasil, 14.

Mocelin, D. G. (2009). Concorrência e alianças entre pesquisadores: reflexões acerca da expansão de grupos de pesquisa dos anos 1990 aos 2000 no Brasil. Revista Brasileira de Pós-Graduação, 6(11), 35-64.

Moreira, N. P., Cunha, N. R. da S., Ferreira, M. A. M., \& Silveira, S. de F. R. (2009). Análise da eficiência relativa dos programas de pós-graduação acadêmicos em Administração, Contabilidade e Turismo. Revista do Serviço Público, 60(4), 365-381.

Siqueira, M. (1988). O tema recursos humanos nas reuniões da ANPAD: trajetórias e perspectivas. Anais do Encontro Nacional da Associação Nacional de Pós-Graduação e Pesquisa em Administração, Foz do Iguaçu, PR, Brasil, 12.

Triola, M. F. (2005). Introdução à estatística (9a ed.). Rio de Janeiro: LTC.

Vergara, S. C., \& Pinto, M. C. S. (2001). Referências teóricas em análise organizacional: um estudo das nacionalidades dos autores referenciados na literatura brasileira. Revista de Administração Contemporânea, 5(Edição Especial), 103-121.

Wood, T., Jr., \& Chueke, G. V. (2008). Ranking de produção científica em administração de empresas no Brasil. Revista de Administração Mackenzie, 9(4), 13-31.

Wood, T., Jr., \& Costa, C. C. de M. (2015). Avaliação do impacto da produção científica de programas selecionados de pós-graduação em Administração por meio do índice $\mathrm{H}$. Revista de Administração, 50(3), 325-337.

Wooldridge, J. M. (2006). Introdução à econometria: uma abordagem moderna. São Paulo: Pioneira Thomson Learning. 